\title{
The Edible Adlay Seed Oil Containing Cinnamaldehyde and Gingerol Prevents DMBA/TPA-Induced Skin Carcinogenesis by Cooperative Ablation of Lipid Rafts
}

\author{
Yaqiu Zheng ${ }^{1}$, Shengnan Geng ${ }^{1}$, Mingjing Meng ${ }^{1}$, Zhenhua Du ${ }^{1}$, Jingjing Yao ${ }^{1}$, Zibo Li ${ }^{1}$, Qun Zhang ${ }^{2}$, Zhenzhen Zhang ${ }^{2}$, Yongjian Duan $^{2}$ and \\ Gangjun Du' ${ }^{1,2 *}$
}

${ }^{1}$ Institute of Pharmacy, Pharmacy College of Henan University, Jinming District, Kaifeng, Henan Province 475004, PR China

${ }^{2}$ Department of Oncology, The First Hospital Affiliated to Henan University, Kaifeng, Henan Province 475001, PR China

\begin{abstract}
It is known that food intake was associated with decreased risk of cancer, we prepared a compounded adlay seed oil (C-ASO) containing $0.5 \%$ cinnamaldehyde and $1 \% 6$-gingerol and evaluated the preventive effects of the oral C-ASO on DMBA/TPA-induced skin carcinogenesis. In contrast to untreated carcinogenic mice, skin tumor incidence and multiplicity were decreased to some extent in single ASO-, cinnamaldehyde- and gingerol-treated mice, whereas the oral C-ASO almost completely reduced susceptibility to skin carcinogenesis by cooperative ablation of enhanced lipid rafts, drastically suppressed phosphatidylinositol 3-Kinases (PI3K)/protein kinases $A$ (Akt) signaling and decreased levels of nuclear transcription factor-kappa B (NF-KB) and cyclin D1 which reduced skin epithelial proliferation and epithelial-mesenchymal transition (EMT) during tumor genesis. This work supports C-ASO as a candidate functional food to prevent carcinogenesis.
\end{abstract}

Keywords: Adlay seed oil, Cinnamaldehyde, Gingerol, Skin carcinogenesis, Lipid rafts

\section{Introduction}

Despite improvements in screening and care, cancer has already become a leading cause of death worldwide [1]. In cancer cells, lipid rafts are viewed as important linchpins, from which signals essential for invasive growth, resistance to death-inducing stimuli and other malignant characteristics are launched [2,3]. Lipid rafts are specialized membrane microdomains of the plasma membrane that is enriched in sphingolipid and cholesterol [4]. Previous study demonstrated that lipid raft disruption results in their internalization dependent on cholesterol [5]. There are quantitative and qualitative raft changes in many cancers due to abnormal accumulation of cholesterol in malignant cells [6], indicating involvement of cholesterol-enriched lipid rafts in initiation and progression of human cancers. Chemoprevention represents the most effective cancer treatment [7], and an alternative strategy to prevent tumor cell is to combine edible composition with health care therapy [8]. Adlay seed has long been used in Asian countries for the treatment of rheumatism, neuralgia, and lung diseases and as a diuretic [9]. The adlay seed oil (ASO) has been shown to reduce blood lipids and have antineoplastic effect [10-12]. Cinnamaldehyde, a major active components of cinnamon which is a spice widely used in cooking since biblical times, has been reported to have anti-obesity and antineoplastic effect [13-15]. 6-gingerol, a major component of fresh ginger which is a commonly used herb and food-flavoring agent, has been reported to inhibit adipogenesis and lipid content accumulation and have also antineoplastic effect [16-18]. Therefore, it is possible that the composition containing three materials might cooperatively ablate lipid rafts to prevent carcinogenesis. To investigate if this hypothesis is correct, we prepared a compounded adlay seed oil (C-ASO) containing $0.5 \%$ cinnamaldehyde and 1\% 6-gingerol and evaluated the preventive effects of the oral C-ASO on 9, 10-dimethylbenzanthracene (DMBA) /12-O-tetradecanolyphorbal13-acetate (TPA) -induced skin carcinogenesis.

\section{Materials and Methods}

\section{Materials}

Adlay seed oil (ASO) was obtained from Guangzhou Masson
Pharmaceutical Ltd. (Guangzhou, China). 12-O-tetradecanolyphorbal13-acetate (TPA) and 9, 10-dimethylbenzanthracene (DMBA) were purchased from Sigma-Aldrich Co. (St. Louis, MO, USA). Cinnamaldehyde and 6-Gingerol (purity $>98 \%$ ) were purchased from $\mathrm{Xi}$-an Helin Biological Engineering Co. (Xi-an, Shanxi, China). Evans blue was purchased from Sigma Chemical Co. (Shanghai, China). The antibodies used include: proliferating cell nuclear antigen (PCNA), Phosphatidylinositol 3-Kinases (PI3K), protein kinases A (Akt), nuclear transcription factor-kappa B (NF- $\mathrm{B}$ ), cyclin D1, E-cadherin, N-cadherin and $\beta$-Actin were obtained from BD Pharmingen. Horseradish peroxidase (HRP) -conjugated goat anti-mouse IgG polyclonal antibody, Peroxidase substrate 3',3'-diaminobenzidine (DAB) and 3-amino-9ethylcarbazole (AEC) were from Nichirei Bioscience (Tokyo, Japan). The mouse quantitative ELISA kits (Tumor necrosis factor- $\alpha$ (TNF- $\alpha$ ), Interleukin-6 (IL-6), High-sensitivity CRP (hs-CRP), reactive oxygen species (ROS), 8-hydroxy-2-deoxyguanosine (8-OHdG)) were obtained from R\&D Systems. Cholesterol kits were obtained from Nanjing Jiancheng Bioengineering Institute (Nanjing, China). Filipin and Alexa 594-cholera toxin B (CTXB) were obtained from Molecular Probes. Standard rodent chow was purchased from Henan Provincial Medical Laboratory Animal Center (Zhengzhou, China). All the other reagents used were the highest purity and commercially available.

\section{Animals}

Cohorts of 5- to 6-wk old female ICR mice were obtained from Henan Provincial Medical Laboratory Animal Center. All mice were

*Corresponding author: Gangjun Du, Department of Oncology, The First Hospital Affiliated to Henan University, Kaifeng, Henan Province 475001, PR China, Tel: +86-371-23880680; E-mail: dyj9062@163.com/724200@henu.edu.cn

Received June 12, 2017; Accepted August 02, 2017; Published August 07, 2017

Citation: Zheng Y, Geng S, Meng M, Du Z, Yao J, et al. (2017) The Edible Adlay Seed Oil Containing Cinnamaldehyde and Gingerol Prevents DMBA/TPA-Induced Skin Carcinogenesis by Cooperative Ablation of Lipid Rafts. J Carcinogene Mutagene 8: 302. doi: 10.4172/2157-2518.1000302

Copyright: @ 2017 Zheng Y, et al. This is an open-access article distributed under the terms of the Creative Commons Attribution License, which permits unrestricted use, distribution, and reproduction in any medium, provided the original author and source are credited. 
housed in individual ventilated cages under a $12 \mathrm{~h}$ light-dark cycles (lights on 7:00 AM to 7:00 PM). The animals were fed standard rodent chow and water. All animal procedures in this study were approved by the Animal Experimentation Ethics Committee of Henan University (the permission number HD20141016), all procedures were performed in strict accordance with the Guide for the Care and Use of Laboratory Animals and the regulation of animal protection committee to minimize the suffering and injury. The animals were monitored daily and euthanized humanely by overdose carbon dioxide at the end of the experiment or the first sign of shortness of breath, reduced locomotion and reduced body weight (greater than $20 \%$ total body weight). All surgery was performed under general anesthesia by intraperitoneal injection of $45 \mathrm{mg} / \mathrm{kg}$ pentobarbital sodium, and all efforts were made to minimize suffering.

\section{DMBA/TPA-induced skin cancer model}

The dorsal skin on the back area of the ICR female mice was shaved 1 day before the commencement of the experiment. The mice were randomly divided into six groups (Normal; DMBA/TPA (control); DMBA/TPA+ASO ( $5 \mathrm{ml} / \mathrm{kg}$ body weight); DMBA/TPA+6-Gingerol (50 mg/kg body weight); DMBA/TPA+Cinnamaldehyde $(25 \mathrm{mg} / \mathrm{kg}$ body weight); DMBA/TPA+C-ASO ( $5 \mathrm{ml} / \mathrm{kg}$ body weight, containing $0.5 \%$ cinnamaldehyde and $1 \% 6$-gingerol), each group included twenty mice. The mice except normal were treated on their shaven backs with topical application of DMBA $(150 \mu \mathrm{g})$ dissolved in $0.2 \mathrm{~mL}$ of acetone once weekly for three times, and the animals in the normal group were treated only with vehicle $(0.2 \mathrm{~mL}$ acetone). One week after initiation with DMBA, the mice except normal were treated topically with TPA (5 $\mu \mathrm{g})$ twice weekly for 10 weeks. Following the first DMBA application, mice were placed on the standard rodent diet and received once daily oral tested compounds for 20 weeks. During the studies, food and water were provided ad libitum, the health of the mice was monitored daily, and body weights were measured weekly. Papillomas appearing on the skin were recorded every week during the experimental period, with only those having diameter $>1 \mathrm{~mm}$ considered as positive. Mice were sacrificed 21 weeks after the first DMBA applications under anesthesia with pentobarbital sodium $(45 \mathrm{mg} / \mathrm{kg})$, skin carcinoma multiplicity (average carcinomas per mouse) were determined, and skins were biochemically processed.

\section{Clinical chemistry}

Blood samples were collected from the orbital venous plexus at sacrifice after $6 \mathrm{~h}$ of fasting for chemical analyses. The serum concentrations of cholesterol, IL-6, hs-CRP and TNF- $a$ were determined according to the manufacturer's protocol.

\section{Oxidative stress analysis}

Serum 8-hydroxy-2'-deoxyguanosine (8-OHdG) levels were measured using an ELISA assay kit. Serum levels of ROS, a marker for oxidative stress, were also determined using an ELISA assay kit.

\section{Immunohistochemistry}

For immunohistochemical (IHC) analyses, serial sections $(5 \mu \mathrm{m})$ of skin were cut and attached to superfrost plus microscope slides. The slides were warmed at $60^{\circ} \mathrm{C}$ for $1 \mathrm{~h}$ and deparaffinized in xylene and rehydrated in decreasing concentrations of ethanol. After blocking with $3 \%$ hydrogen peroxide for $10 \mathrm{~min}$, the sections were incubated with $0.1 \%$ TritonX-100 in phosphate-buffered saline (PBS) for 20 min at room temperature and next immersed in a blocking solution of $5 \%$ bovine serum albumin (BSA) for $20 \mathrm{~min}$ at room temperature. The sections were incubated with primary antibodies (1:100) against PCNA, E-cadherin and $\mathrm{N}$-cadherin overnight at $4^{\circ} \mathrm{C}$. Antigenantibody binding was detected by using Dako Cytomation labeled streptavidin biotin (LSAB) System-HRP kit. The signal was developed with the peroxidase substrate $\mathrm{DAB}$ which appears as a brown reaction product or with AEC which appears as a red reaction product. All sections were counterstained with hematoxylin and were imaged under a microscope. A semi-quantitative histological score (HSCORE) was used as described previously.

\section{Lipid raft detection}

Skin single cell suspensions of were prepared by collagenase IV digestion. The cells were attached to superfrost plus microscope slides and were fixed with $3.7 \%$ paraformaldehyde in PBS at $4^{\circ} \mathrm{C}$ for $20 \mathrm{~min}$, rinsed with PBS, and treated with $1.5 \mathrm{mg} / \mathrm{ml}$ glycine in PBS to quench free aldehyde group. Cells were then stained with filipin $(0.05 \mathrm{mg} / \mathrm{ml})$ and Alexa 594-CTXB $(0.04 \mu \mathrm{g} / \mathrm{ml})$ for plasma membrane cholesterol and monosialotetrahexosylganglioside (GM1) staining, respectively. Cells were visualized using confocal imaging with a ZEISS LSM 510 Meta/UV.

\section{Western blot analysis}

Skin extracts were prepared in immunoprecipitation cell lysis buffer and an equal amount of protein was separated on $12 \%$ sodium dodecyl sulfate-polyacrylamide gel electrophoresis, electroblotted on nitrocellulose membranes and probed with specific antibodies against PI3K, Akt, NF- $k B$, cyclin D1 and GAPDH. Antibody binding was detected by enhanced pico chemiluminescence (Pierce, Rockford, IL).

\section{Statistical analyses}

The measures are presented as mean \pm SD and were statistically analyzed using the GraphPad Prism software program, Version 5.0 (San Diego, CA, USA). One-way analysis of variance (ANOVA) was used to compare groups by the Mann-Whitney $U$ test. The differences were considered significant when the two-sided $\mathrm{P}$ value was less than 0.05 .

\section{Results}

\section{C-ASO almost completely reduced susceptibility to skin carcinogenesis}

Natural phytochemicals derived from dietary sources have gained significant recognition in the potential management of several human clinical conditions [19], and the combination of dietary phytochemicals are considered more promising for higher efficacy [20]. In this study, a two-stage skin carcinogenesis experiment was conducted. One week after initiation with DMBA, the mice were treated topically with TPA for 10 weeks. Following the first DMBA application, mice received once daily oral tested compounds for 20 weeks. Tumor incidence and multiplicity were calculated after mice were sacrificed at 21 weeks. Under these experimental conditions, oral treatment of ASO, cinnamaldehyde and gingerol alone or combined had no effect on body weight, whereas C-ASO led to lean body phenotypes (<fifteen percent of normal body weight) (Figure 1A). In contrast to untreated carcinogenic mice, skin tumor incidence and multiplicity were decreased to some extent in single ASO-treated, cinnamaldehyde-treated and gingerol-treated mice. However, C-ASO almost completely reduced susceptibility to skin carcinogenesis (Figure 1B and C). 
A

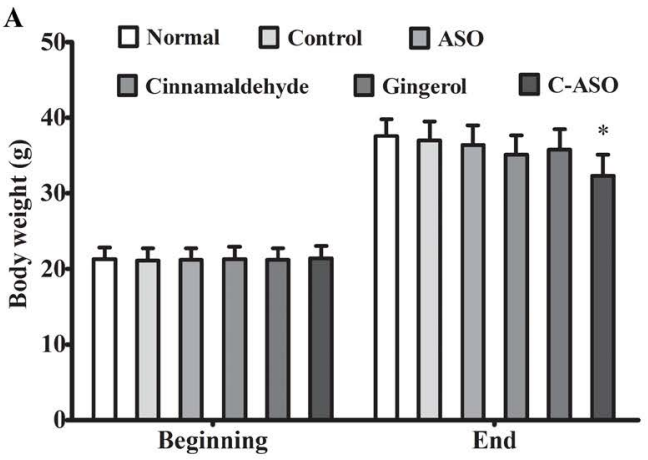

$\mathbf{C}$

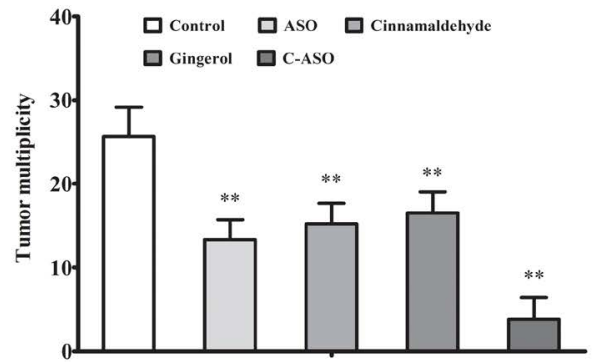

B

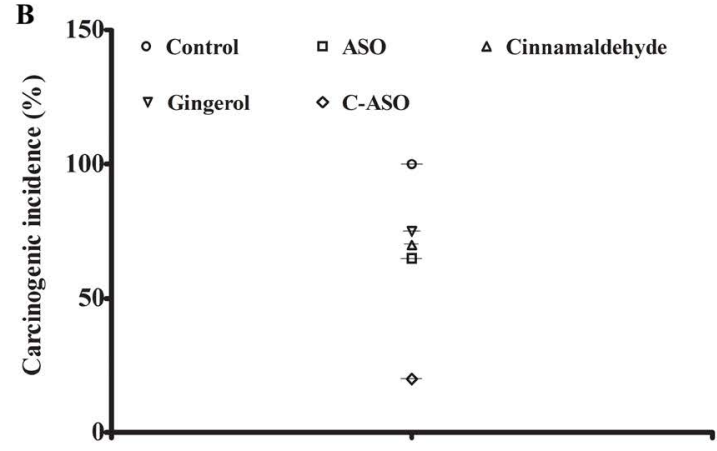

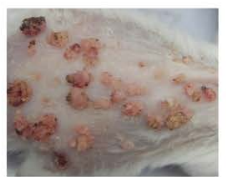

Control

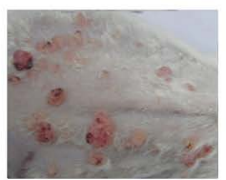

Gingerol

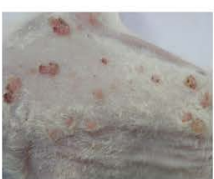

ASO

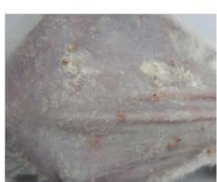

C-ASO

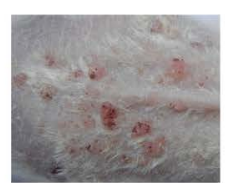

Cinnamaldehyde

Figure 1: C-ASO synergistically prevented DMBA/TPA-induced skin carcinogenesis. The mice were treated on their shaven backs with topical application of DMBA once weekly for three times, one week after initiation with DMBA, the mice were treated topically with TPA twice weekly for 10 weeks. Following the first DMBA application, mice received once daily oral tested compounds for 20 weeks. ASO, cinnamaldehyde and gingerol alone or combined had no effect on body weight, whereas C-ASO led to lean body phenotypes $(A)(n=20)$. Skin carcinoma incidence (B) and multiplicity (C) were decreased to some extent in single ASO-treated, cinnamaldehyde-treated and gingerol-treated mice, and C-ASO almost completely reduced susceptibility to skin carcinogenesis ( $\mathrm{n}=20$ ). The data present Mean \pm SD and statistical significance was determined by the Mann-Whitney $U$ test. ${ }^{*} P<0.05,{ }^{* *} P<0.01$ vs. control.

\section{C-ASO synergistically decreased inflammation and oxidative stress during carcinogenesis}

To further verify above results, we carried out clinical chemistry test and oxidative stress analysis at 21 weeks. Consistent with above results, serum levels of IL-6, hs-CRP and TNF- $a$ were decreased to some extent in single ASO-treated, cinnamaldehyde-treated and gingerol-treated mice but were very significantly suppressed by C-ASO compared to untreated carcinogenic mice (Figure 2A-C). Similarly, serum levels of 8-OHdG and ROS were only decreased to some extent in single ASOtreated, cinnamaldehyde-treated and gingerol-treated mice but were very significantly suppressed by C-ASO (Figure $2 \mathrm{D}$ and $\mathrm{E}$ ).

\section{C-ASO synergistically decreased skin cell lipid rafts and suppressed cellular signaling during carcinogenesis}

To explore why C-ASO synergistically reduced susceptibility to skin carcinogenesis, we examined DMBA/TPA-associated changes in serm cholesterol, cell membrane lipid rafts by plasma membrane cholesterol and GM1 staining, and cellular signaling such as PI3K, Akt, NF- $\mathrm{kB}$ and cyclin D1 by western blot analyses at 21 weeks. As shown in Figure 3A, serum levels of IL-6 were decreased to some extent in single ASO-treated, cinnamaldehyde-treated and gingerol-treated mice but were very significantly suppressed by C-ASO compared to untreated carcinogenic mice. Consistently, the skin cell lipid rafts were decreased to some extent in single ASO-treated, cinnamaldehyde-treated and gingerol-treated mice but were very significantly suppressed by C-ASO compared to untreated carcinogenic mice (Figure 3B and C). Similarly, the skin protein expression of PI3K, Akt, NF- $\kappa \mathrm{B}$ and cyclin D1 was only decreased to some extent in single ASO-treated, cinnamaldehydetreated and gingerol -treated mice but were very significantly suppressed by C-ASO (Figure 3D).

\section{C-ASO synergistically decreased skin epithelial proliferation and epithelial-mesenchymal transition (EMT)}

Carcinogenesis is the final results of epithelial proliferation and EMT [21]. To verify the synergistical effect of C-ASO on cancer prevention, we also examined DMBA/TPA-associated changes in skin epithelial proliferation and EMT by immunohistochemistry at 21 weeks. Consistent with above results, PCNA, a skin epidermal proliferation marker, were decreased to some extent in single ASOtreated, cinnamaldehyde-treated and gingerol-treated mice but were very significantly suppressed by C-ASO compared to untreated carcinogenic mice (Figure 4A and B). Similarly, lung EMT indicated by E-cadherin and N-cadherin were only decreased to some extent in single ASO-treated, cinnamaldehyde-treated and gingerol-treated mice but were very significantly suppressed by C-ASO (Figure 4A and B).

\section{Discussion}

The typical foods and spices containing various phytochemicals have been used for prevention and treatment of a variety of human ailments since time immemorial [22], there has been growing interest 

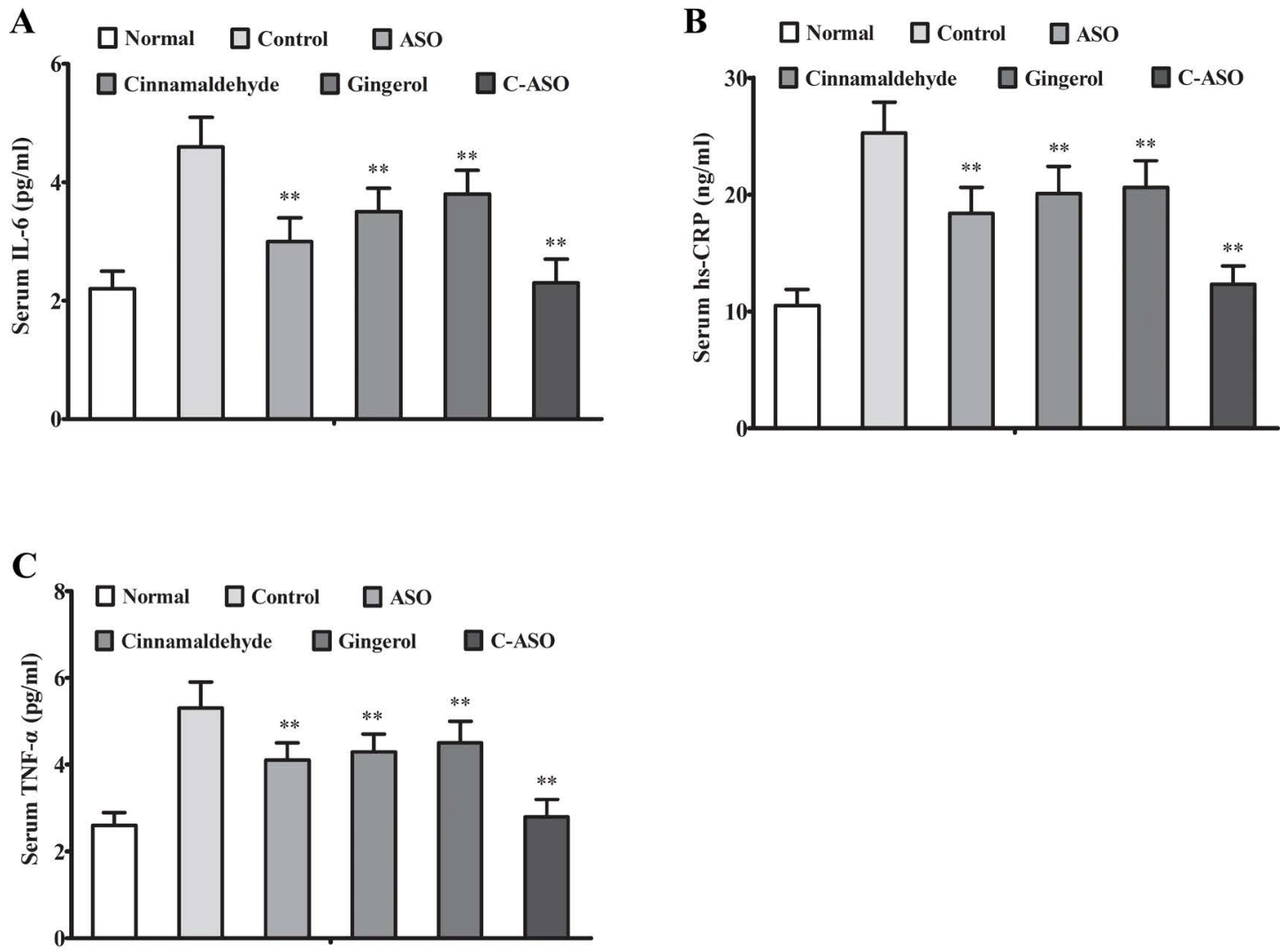

D

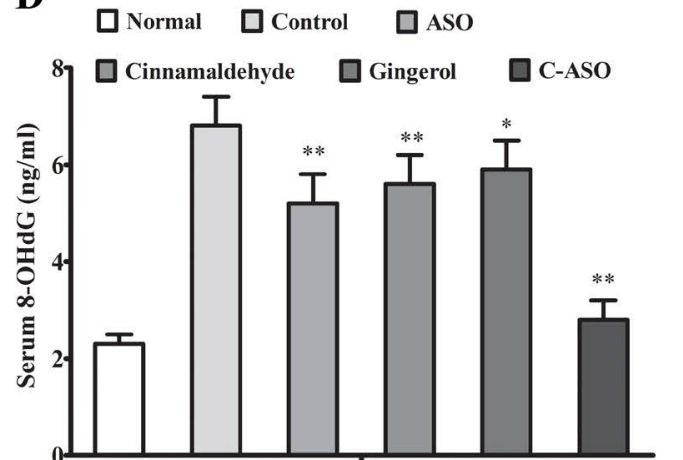

$\mathbf{E}$

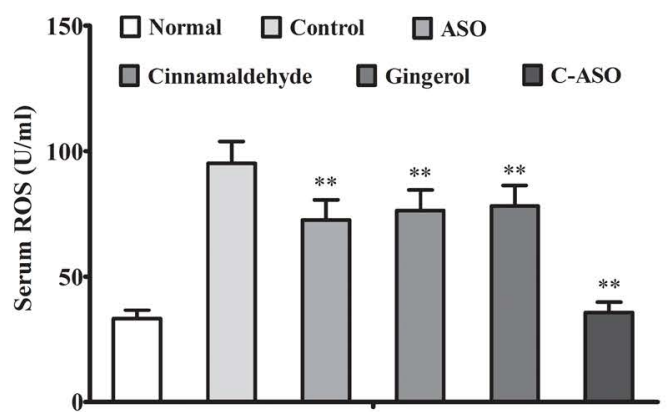

Figure 2: C-ASO synergistically decreased inflammation and oxidative stress during carcinogenesis. C-ASO synergistically decreased inflammation indicated by increased serum levels of IL-6 (A), hs-CRP (B), TNF- $\alpha(C)(n=5)$. C-ASO synergistically decreased oxidative stress indicated by increased serum levels of 8-OHdG (D) and $\operatorname{ROS}(E)(n=5)$. The data present Mean $\pm S D$ and statistical significance was determined by the Mann-Whitney $U$ test. ${ }^{*} P<0.05$, ${ }^{* *} P<0.01$ vs. control.

in cancer prevention by food plants and their products in recent years [23]. Adlay seed is a kind of nourishing food which has also been used in traditional Chinese medicine [24], and ASO has been proved to reduce the abdominal fat tissue and increase the total antioxidant capacity in hyper-lipidemic rats [25]. In addition, a pharmaceutical grade extract of adlay seed is currently the most commonly used treatment for cancer in China [26]. Although clinical data also support the use of coix seed and its extracts for cancer treatment [27], a single agent based treatments coax seed and its extracts may not always be sufficient to provide chemo preventive efficacy, therefore, the new concept of combination chemoprevention by multiple agents has become an increasingly attractive area of study. In this study, we showed that C-ASO could reduce susceptibility to skin carcinogenesis with higher efficacy.

Targeting malfunctioning molecules along the disrupted signal transduction pathway in cancer represent a rational strategy in chemoprevention [28]. A wide number of signal transduction processes related to cell adhesion, migration, as well as to cell survival and proliferation, which play major roles in cancer development and progression, are dependent on lipid rafts $[29,30]$. In this study, we 
A
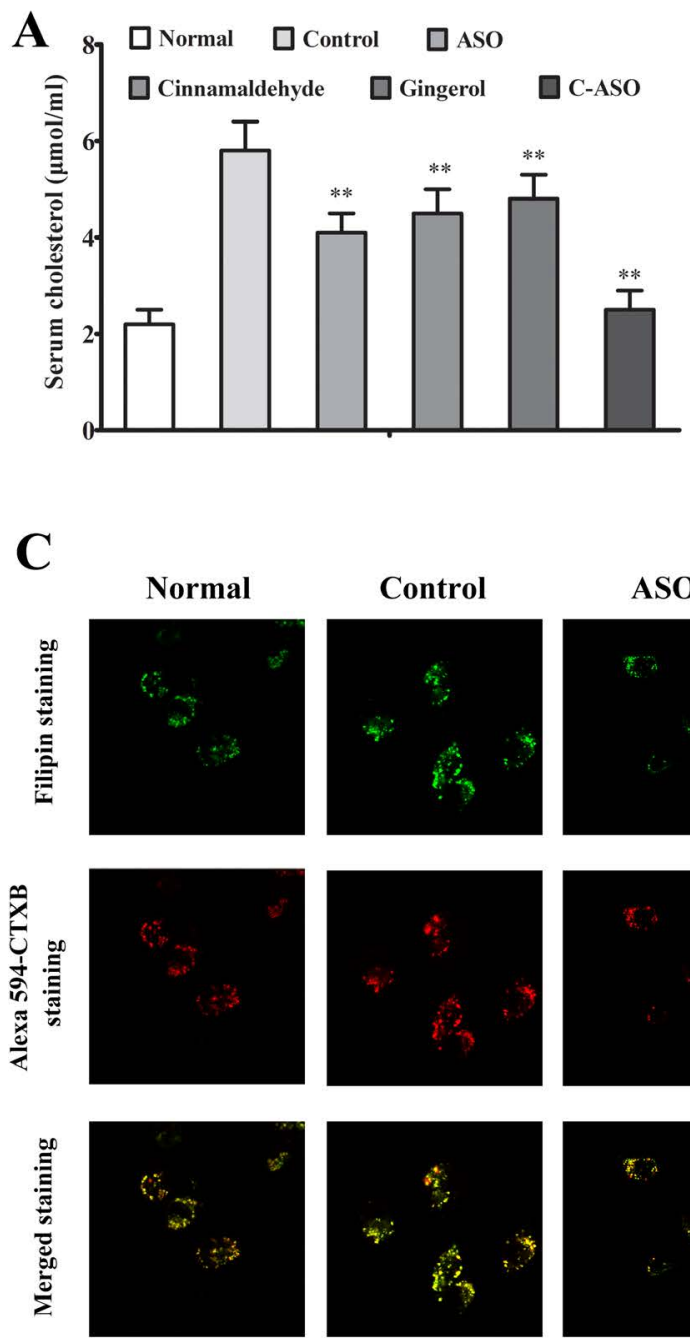

\section{Control}
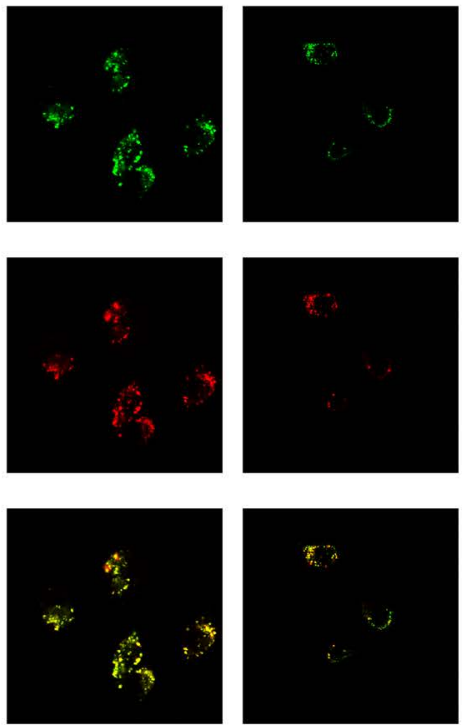

ASO

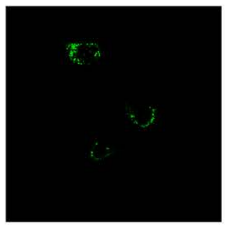

B

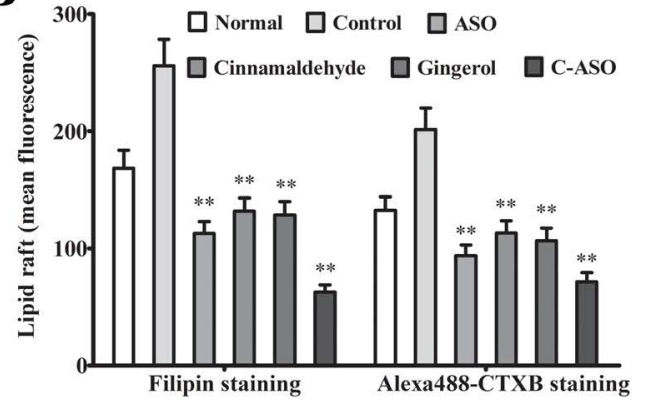

D

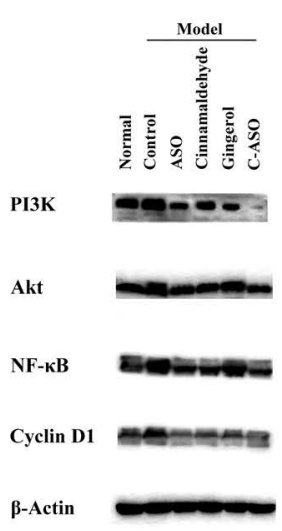

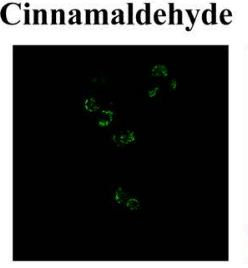
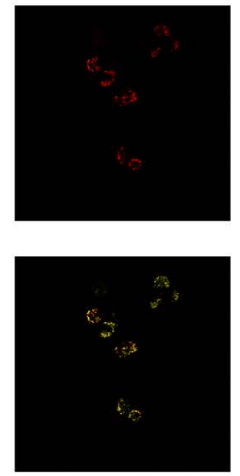

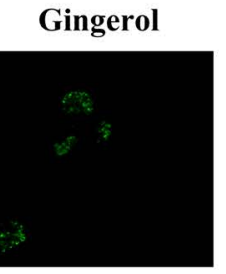

C-ASO
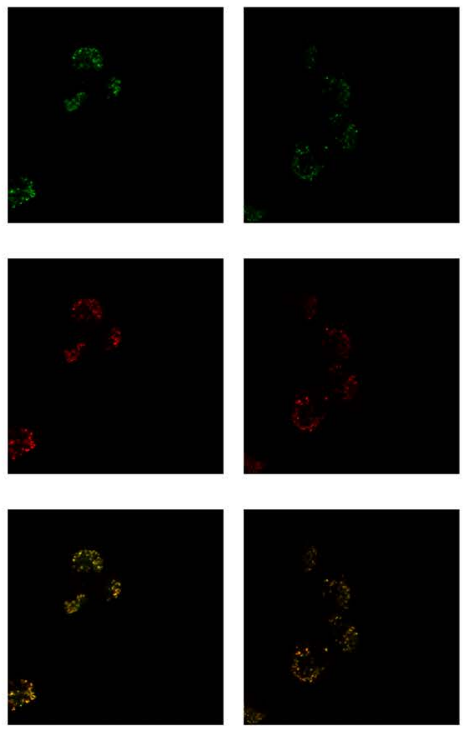

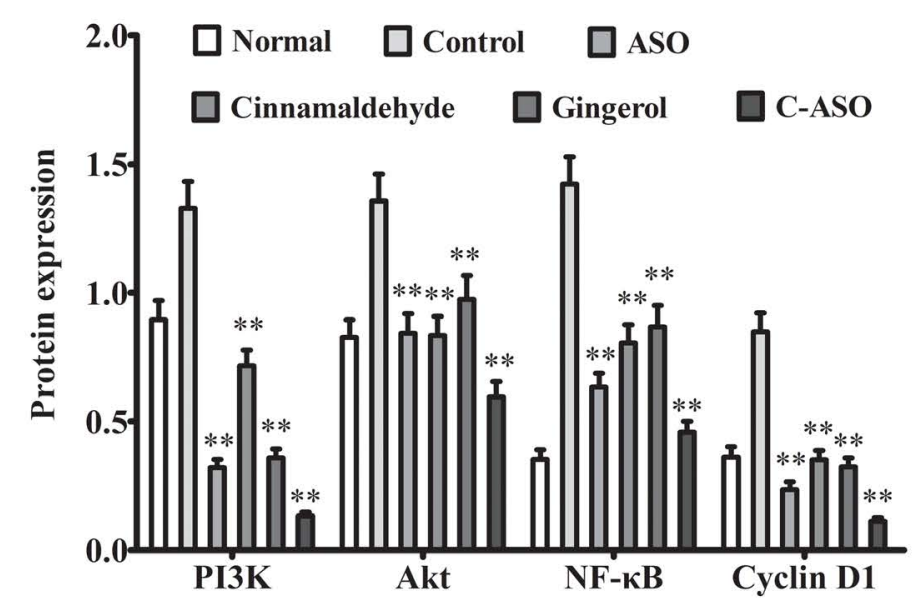

Figure 3: C-ASO synergistically decreased skin cell lipid rafts and suppressed cellular signaling during carcinogenesis. C-ASO synergistically decreased serum cholesterol (A) and skin cell lipid rafts (B and C), and suppressed protein expression of PI3K, Akt, NF-kB and cyclin D1 examined by western blot (D). The data present Mean \pm SD of triplicate assays and statistical significance was determined by the Mann-Whitney $U$ test. ${ }^{*} P<0.05$, ${ }^{* *} P<0.01$ vs. control. 

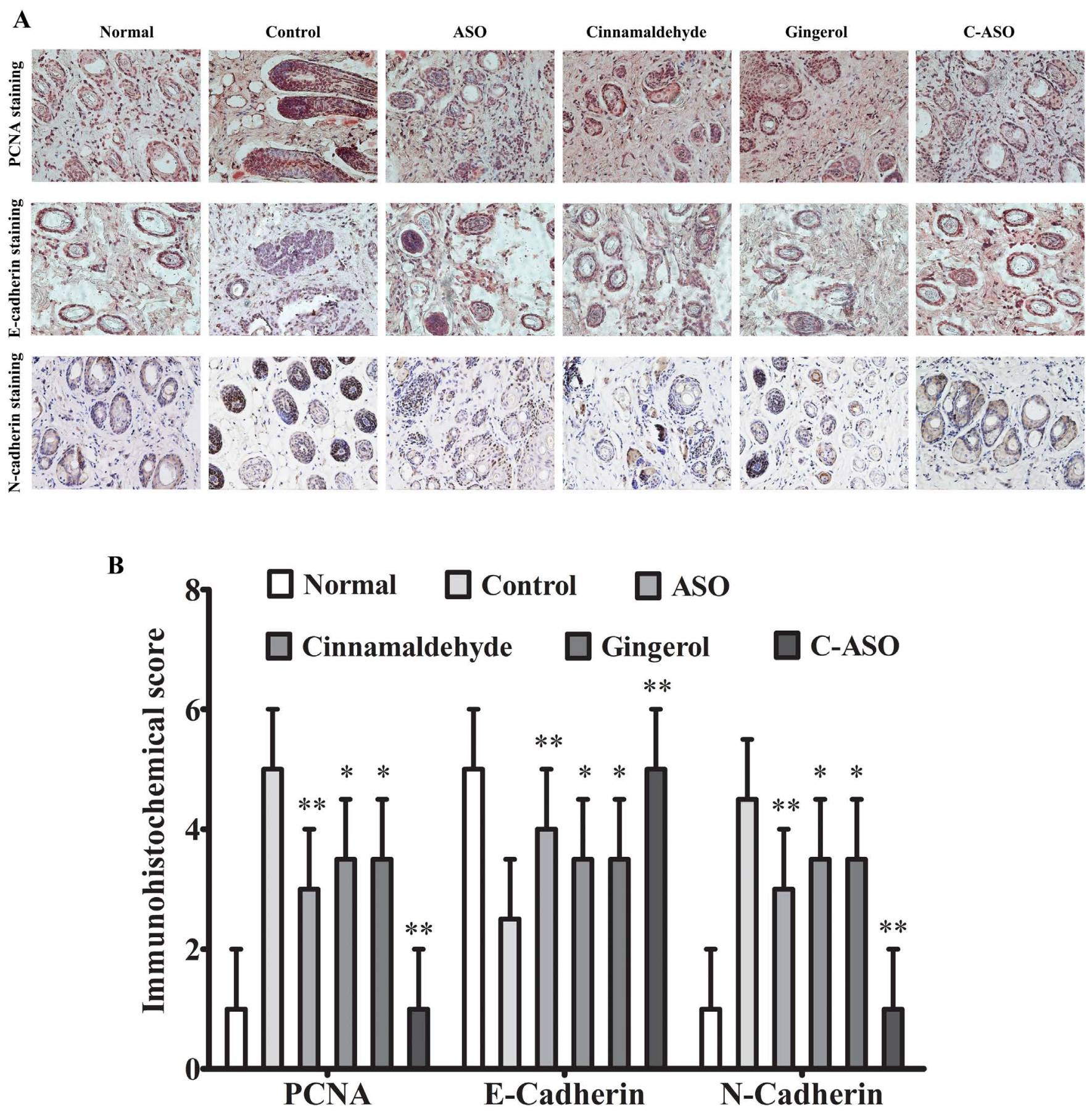

Figure 4: C-ASO synergistically prevented skin epithelial proliferation and EMT during carcinogenesis examined by immunohistochemistry. (A) C-ASO synergistically prevented skin epithelial proliferation indicated by increased PCNA and EMT indicated by decreased E-cadherin and increased N-cadherin. (B) The semi-quantitative histological score. Images were taken at $\times 40$ magnification. The data present Mean \pm SD of triplicate assays and statistical significance was determined by the MannWhitney $U$ test. ${ }^{*} \mathrm{P}<0.05$, ${ }^{* *} \mathrm{P}<0.01$ vs. control.

firstly supposed that the combination of dietary lipid-reducing agents could target lipid rafts to prevent cell signaling cascades, we found that C-ASO synergistically decreased inflammation and oxidative stress during carcinogenesis, indicating a possible endeavor in cancer chemoprevention because chronic inflammation is associated with increased risk of cancer. Next, we showed that C-ASO synergistically decreased skin cell rafts and suppressed PI3K/Akt, NF- $\kappa B$ and cyclin
D1 signaling during carcinogenesis, indicating a lipid raft disruption and an abortion of cell signaling cascades. Finally, we showed that C-ASO synergistically decreased skin epithelial proliferation and EMT during carcinogenesis, indicating the final result induced by C-ASO.

Early interruption of the carcinogenic process is a priority strategy to reduce the burden of cancer; however, the intervention may be 
ineffective if pre-neoplastic lesions with cellular changes cannot be reversed [31]. Recent research efforts have been greatly emphasized on the recognition of naturally occurring plant derived substances that are capable of inhibiting, retarding or reversing the development of cancer [32], and comparative effectiveness research designs and the value of information obtained from large-scale prevention studies are necessary in order for preventive interventions to become a routine part of cancer management [33]. Our results opened up a new avenue for cancer chemoprevention and suggested that C-ASO is promising and inexpensive in this endeavor.

\section{Acknowledgments}

This study was supported by National Natural Science Foundation of China (No. 81173094, 81472745).

\section{References}

1. Marmot MG, Altman DG, Cameron DA, Dewar JA (2013) The benefits and harms of breast cancer screening: an independent review. $\mathrm{Br} \mathrm{J}$ Cancer 108 2205-2240.

2. Paillasses MR, de Medina P, Amouroux G, Mhamdi L (2009) Signaling through cholesterol esterification: a new pathway for the cholecystokinin 2 receptor involved in cell growth and invasion. J Lipid Res 50: 2203-2211.

3. George KS, Wu S (2012) Lipid Raft: A Floating Island of Death or Survival. Toxicol Appl Pharmacol 259: 311-319.

4. Fantini J, Yahi N (2010) Molecular insights into amyloid regulation by membrane cholesterol and sphingolipids: common mechanisms in neurodegenerative diseases. Expert Rev Mol Med 12: e27.

5. McHenry AJ, Sciacca MFM, Brender JR, Ramamoorthy A (2012) Does cholesterol suppress the antimicrobial peptide induced disruption of lipid raft containing membranes? Biochim Biophys Acta 1818: 3019-3024.

6. Baenke F, Peck B, Miess H, Schulze A (2013) Hooked on fat: the role of lipid synthesis in cancer metabolism and tumour development. Dis Model Mech 6: $1353-1363$.

7. Keith RL, Miller YE (2013) Lung cancer chemoprevention: current status and future prospects. Nat Rev Clin Oncol 10: 334-343.

8. Kris-Etherton PM, Akabas SR, Bales CW, Bistrian B (2014) The need to advance nutrition education in the training of health care professionals and recommended research to evaluate implementation and effectiveness1,2,3,4. Am J Clin Nutr 99: 1153S-1166S

9. Kuo CC, Chen HH, Chiang W (2012) Adlay is a Potential Cancer Chemopreventive Agent toward Multistage Carcinogenesis Processes. J Tradit Complement Med 2: 267-275.

10. Lu X, Liu W, Wu J, Li M (2013) A polysaccharide fraction of adlay seed (Coixlachryma-jobi L.) induces apoptosis in human non-small cell lungcancer A549 cells. Biochem Biophys Res Commun 430: 846-851.

11. Son BK, Kim JY, Lee SS (2008) Effect of adlay, buckwheat and barley on lipid metabolism and aorta histopathology in rats fed an obesogenic diet. Ann Nutr Metab 52: 181-187.

12. Manosroi A, Sainakham M, Chankhampan C, Manosroi W, Manosroi J (2016) In vitro anti-cancer activities of Job's tears (Coix lachryma-jobi Linn.) extracts on human colon adenocarcinoma. Saudi J Biol Sci 23: 248-256.

13. Kwon HK, Hwang JS, So JS, Lee CG (2010) Cinnamon extract induces tumor cell death through inhibition of NFKB and AP1. BMC Cancer 10: 392

14. Camacho S, Michlig S, de Senarclens-Bezençon C, Meylan J (2015) AntiObesity and Anti-Hyperglycemic Effects of Cinnamaldehyde via altered Ghrelin Secretion and Functional impact on Food Intake and Gastric Emptying. Sci Rep 5: 7919 .
15. Wani KD, Kadu BS, Mansara P, Gupta P (2014) Synthesis, Characterization and In Vitro Study of Biocompatible Cinnamaldehyde Functionalized Magnetite Nanoparticles (CPGF Nps) For Hyperthermia and Drug Delivery Applications in Breast Cancer. PLoS One 9: e107315.

16. Li M, Chen PZ, Yue QX, Li JQ (2013) Pungent ginger components modulates human cytochrome P450 enzymes in vitro. Acta Pharmacol Sin 34: 1237-1242.

17. Park H, Hwang YH, Kim DG, Jeon J, Ma JY (2015) Hepatoprotective Effect of Herb Formula KIOM2012H against Nonalcoholic Fatty Liver Disease. Nutrients 7: 2440-2455

18. Lv L, Chen H, Soroka D, Chen X (2012) 6-Gingerdiols as the Major Metabolites of 6-Gingerol in Cancer Cells and in Mice and Their Cytotoxic Effects on Human Cancer Cells. J Agric Food Chem 60: 11372-11377.

19. Lee J, Jo DG, Park D, Chung HY, Mattson MP (2014) Adaptive Cellular Stress Pathways as Therapeutic Targets of Dietary Phytochemicals: Focus on the Nervous System. Pharmacol Rev 66: 815-868.

20. Lee JH, Khor TO, Shu L, Su ZY (2013) Dietary phytochemicals and cance prevention: Nrf2 signaling, epigenetics, and cell death mechanisms in blocking cancer initiation and progression. Pharmacol Ther 137: 153-171.

21. Tiwari N, Meyer-Schaller N, Arnold P, Antoniadis H (2013) Klf4 Is a Transcriptional Regulator of Genes Critical for EMT, Including Jnk1 (Mapk8) PLoS One 8: e57329.

22. Koppikar SJ, Choudhari AS, Suryavanshi SA, Kumari S (2010) Aqueous Cinnamon Extract (ACE-c) from the bark of Cinnamomum cassiacauses apoptosis in human cervical cancer cell line $(\mathrm{SiHa})$ through loss of mitochondrial membrane potential. BMC Cancer 10: 210.

23. Chun KS, Kundu J, Chae IG, Kundu JK (2014) Carnosol: A Phenolic Diterpene With Cancer Chemopreventive Potential. J Cancer Prev 19: 103-110.

24. Yang RS, Lu YH, Chiang W, Liu SH (2013) Osteoporosis Prevention by Adlay (Yì Yĩ: The Seeds of Coix Lachryma-Jobi L. var. ma-yuen Stapf) in a Mouse Model. J Tradit Complement Med 3: 134-138.

25. Yu F, Gao J, Zeng Y, Liu CX (2011) Effects of adlay seed oil on blood lipids and antioxidant capacity in hyperlipidemic rats. J Sci Food Agric 91: 1843-1848.

26. Yang X, Hao J, Zhu CH, Niu YY (2015) Survival Benefits of Western and Traditional Chinese Medicine Treatment for Patients with Pancreatic Cancer Medicine 94: e1008.

27. Qu D, He J, Liu C, Zhou J, Chen Y (2014) Triterpene-loaded microemulsion using Coix lacryma-jobi seed extract as oil phase for enhanced antitumor efficacy: preparation and in vivo evaluation. Int J Nanomedicine 9: 109-119.

28. Davis NM, Sokolosky M, Stadelman K, Abrams SL (2014) Deregulation of the EGFR/ PI3K/ PTEN/ Akt/mTORC1 pathway in breast cancer: possibilities for therapeutic intervention. Oncotarget 5: 4603-4650.

29. Sun Y, Guo W, Ren T, Liang W (2014) Gli1 inhibition suppressed cell growth and cell cycle progression and induced apoptosis as well as autophagy depending on ERK1/2 activity in human chondrosarcoma cells. Cell Death Dis 5: e979.

30. Bieberich E (2012) It's a lipid's world: Bioactive lipid metabolism and signaling in neural stem cell differentiation. Neurochem Res 37: 1208-1229.

31. Meyskens FL, Mukhtar H, Rock CL, Cuzick J (2015) Cancer Prevention: Obstacles, Challenges and the Road Ahead. J Natl Cancer Inst 8: 108.

32. Vyas AR, Singh SV (2014) Molecular targets and mechanisms of cancer prevention and treatment by withaferin a, a naturally occurringsteroidal lactone. AAPS J 16: 1-10.

33. Harris JR, Brown PK, Coughlin S, Fernandez ME (2005) The Cancer Prevention and Control Research Network. Prev Chronic Dis 2: A21. 